\title{
Henri Poincaré and algebraic geometry
}

\author{
Ciro Ciliberto
}

Published online: 16 May 2013

(c) Centro P.RI.ST.EM, Università Commerciale Luigi Bocconi 2013

\begin{abstract}
The paper presents an overview of Poincaré's main contributions to algebraic and arithmetic geometry, putting them in perspective.
\end{abstract}

\section{Mathematics Subject Classification $\quad 01 \mathrm{~A} 55 \cdot 01 \mathrm{~A} 60$}

\section{Introduction}

During a visit to Rome in 1925, a young André Weil (1906-1998) asked Francesco Severi (1879-1961) for an opinion on Salomon Lefschetz (1884-1972). Here's the needle-sharp answer, as Weil reports:

É bravo. Non è un Poincaré. Poincaré è un'aquila. Lefschetz è un passero, è bravo però, è bravo.

$\mathrm{He}$ is good. He is no Poincaré. Poincaré is an eagle, Lefschetz is a sparrow, but he's good, he's good [32, p. 43] (his italics).

Severi, one of the fathers of the so-called Italian School of algebraic geometry, was certainly harsh in judging his peers. He was also inclined to play down the scientific achievements of others and emphasise his own. Hence it is likely that he was keener to praise Henri Poincaré (1854-1912), who had passed away just over a decade earlier, and with whom any comparison made no longer sense, rather than acknowledge the remarkable skills of Lefschetz, who was just stepping into the spotlight as the successor to Émile Picard (1856-1941) and to Poincaré. On the other hand, given that Lefschetz is one of the most important and

C. Ciliberto $(\square)$

Dipartimento di Matematica, Università di Roma Tor Vergata,

Via della Ricerca Scientifica, 00133 Roma, Italy

e-mail: cilibert@axp.mat.uniroma2.it influential mathematicians of the twentieth century, we should interpret Severi's words as a well-deserved tribute to Poincaré, more than as a criticism to Lefschetz.

Poincaré was a real polymath, and not many scientists, let alone mathematicians, could stand up to him. No peak was beyond his reach. His eagle eye scanned broad landscapes which others only managed to glimpse partially, thus transcending his contemporaries' understanding. That is how he spotted significant details, and then dived on his preywith killer precision - by formulating the right theory for solving problems that had been open for years, sometimes with incredibly avant-garde methods. Or, with a few nods, he would hint at pioneering lines of enquiry that, in hindsight, can be seen to have kept generations of scholars busy. What strikes even the casual reader of his mathematical work is the unrivalled ability to excel in several genres. This versatility made him hardly just a philosopher, a physicist, a mathematician, or merely an analyst, a geometer, an algebraist, a logician and a number theorist. Poincaré was extraordinary in that he was all these in one figure, and one of the highest calibre. The awesome aspect is that they coexisted in him. It is not hard to find in his writings sentences like (Fig. 1):

Ainsi se trouve démontré, par des méthodes purement arithmétiques, ce théorème si utile dans la théorie def fonctions abéliennes, ce qui prouve une fois de plus que l'analiste ne saurait se passer du secours de la théorie des nombres [20, p. 143].

Thus it is demonstrated, with purely arithmetic methods, this very useful theorem in the theory of Abelian functions, proving once more that the analyst cannot avoid resorting to number theory (my italics).

It goes without saying that much has already been said to try to capture his persona. I personally do not know if 


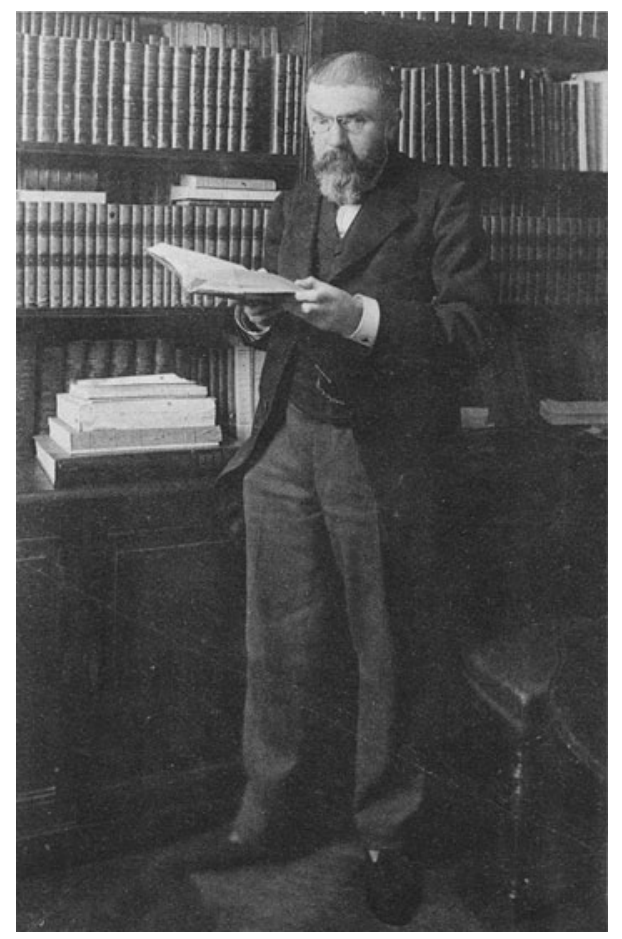

Fig. 1 Henri Poincaré in his library, 1908. Reproduced by generous permission of the LHSP-Archives Henri-Poincaré. UMR 7117 CNRS/Université de Lorraine

every single facet of his work has been clarified, or whether all his ideas have been developed to do him justice. But it is a fact that very few mathematicians have been cited, mentioned or studied more extensively.

Given these premises, to speak of Poincaré and say something original becomes a preposterous undertaking, actually a losing battle. This is true especially if one expects to take on only one aspect of his whole production. No one eludes predefined schemes and ill-fits narrow classifications more than Poincaré. In him any separation between the various domains of human knowledge, every boundary line between branches of mathematics in particular, dissolve, only to leave the reader with a sense of wonder for his exceptionally wide-ranging view on things.

As I was collecting my thoughts for this survey article, I went through what, for me, are his greatest contributions to algebraic geometry, trying to make a list. I was acquainted with Phillip Griffiths's nice paper [11], so I chose it as guide for the task. I recommend its first five or six pages even to the non-expert, for they contain a short review of Poincarés legacy in algebraic geometry and mention some of their most important developments. I was aware that Poincaré, shortly before his untimely death, had written a long memoir [31] for the journal Acta Mathematica, at the request of Gösta MittagLeffler (1846-1927), in which he recollected all his works and included several detailed comments. This report came out posthumously, 9 years after the author had died, and contains no mention of what is considered one of Poincaré's primary contributions to algebraic geometry (and mathematics as a whole): the so-called fundamental theorem of irregular surfaces, which he proved after finishing [31]. At any rate, I started from these references and prepared a (certainly incomplete) inventory of fifty or so main articles related to algebraic geometry, as we nowadays call it. Then I begun to read them, an exercise far from easy, given that Poincaré, in the true spirit of his times, did not write in the standard statement-then-proof combination (this is a heritage of Bourbakism). Rather, his arguments have to be followed to the very end in order to grasp what is actually claimed, what is proved, and what conjectured. Needless to say, I was able to go deeply into a few items only. Still, that was enough for the two purposes I had. First, I managed to experience the qualities mentioned above, and secondly, I became eager to read his work more thoroughly.

\section{How interested was Poincaré in algebraic geometry?}

At the beginning of [11] Griffiths says (my italics):

Although the subject of algebraic geometry was not one of Poincaré's major preoccupations, his work in the field showed characteristic insight and brilliance and certainly has had a lasting effect.

Even though I definitely agree with the latter part of the statement, I do have some doubts on the former. Griffiths's point seems to be confirmed by Poincaré himself, who in [31] never employs the words algebraic geometry. But we must remember that this term, as we understand it today, was probably not so common at the time.

I do not know, and it is probably arbitrary to say, what Poincaré's main concern was, given the enormous spectrum of his interests. What is undeniable is that his early scientific writings dealt with topics that we currently label as belonging to the field of algebraic geometry. His first articles, on quadratic and cubic forms over the rationals or other number fields, dated 1880, and would be classified today under arithmetic geometry, the framework of Andrew Wiles's proof of Fermat's last theorem. In 1881 begins the extensive series of papers on classical Fuchsian functions, which have to do with the uniformisation of varieties acted upon by discrete groups, and with the study of modular forms. In 1882 appears the first in an equally long sequence of works (some in collaboration with Picard) on Abelian functions. The first, short but brilliant, report on residues of differential forms saw the light in 1886, followed the next year by an extended version with complete details.

In these papers, all more or less written during the first 10 years of his career, Poincaré had already touched upon every algebro-geometrical topic that he will sooner or later 
contribute to the most. So, whether preoccupied or not, Poincaré dwelt on algebraic geometry already at an early stage, although, strictly speaking, he might not be branded-as Griffiths certainly meant-an algebraic geometer.

\section{Algebraic geometry during Poincaré's time}

What exactly was algebraic geometry at the end of the 1870s, when Poincaré made his debut on the stage of mathematical research? The latter, not so differently from today, was articulated in three main areas.

\subsection{Analysis}

The focus was on algebraic functions, and their integrals, called Abelian integrals after the pivotal work of Nils Abel (1802-1829). Roughly put, the subject goes back to Leonard Euler (1707-1783), who studied elliptic functions, then Giulio de' Toschi, count of Fagnano (1682-1766), and Adrien-Marie Legendre (1752-1833). But it is in the nineteenth century that the matter reaches its full maturity under Abel, Carl Gustav Jacobi (1804-1851) and Bernhard Riemann (1826-1866). I will not add more at this juncture, and instead suggest my 2008 article [5] to the interested reader. Let me just say that the long and involved climb up this vertiginous mathematical peak, only apparently completed by Riemann, had disclosed to the mathematicians who managed to conquer it new and more fascinating endeavours. That territory would soon be charted by people of the calibre of Karl Weierstrass (1815-1897), Sonia Kovalevskaya (1850-1891), Émile Picard, and Poincaré himself.

\subsection{Arithmetic}

The object of concern was polynomial equations with coefficients in the integers (Diophantine equations), the rationals, or their algebraic extensions (the number fields). This area went back a long way, and had recently witnessed an impressive advancement due to Legendre and Carl Friedrich Gauss (1777-1855). As fundamental as the results of these people are, they were clearly not the definitive answer to an immense number of open problems. On the contrary, the landscape had just started to unveil itself, and was also explored by Poincaré's contemporaries Leopold Kronecker (1823-1891), Ernst Eduard Kummer (1810-1893) and Julius Dedekind (1831-1916).

\subsection{Geometry}

Of the three, this was the youngest branch of study, one still in its infancy at the time. It had its roots in Gaspard Monge's (1746-1818) projective geometry, developed between the end of the eighteenth and the beginning of the nineteenth centuries. The discipline became all the more important only later, with Jean-Victor Poncelet (1788-1867), Karl von Staudt (1798-1867), Jakob Steiner (1796-1863) and Julius Plücker (1801-1868). We owe the projective jargon, deployed to tackle algebraic problems and construe and simplify Riemann's approach to Abelian integrals, to Luigi Cremona (1830-1903) in the 1860-70s, and in the same years to Riemann's student Alfred Clebsch (1833-1872) (see the first part of [2] for more on this story). Clebsch's work was carried on by Paul Albert Gordan (1837-1912), Alexander von Brill (1842-1935) and Max Noether (1844-1922). The latter two, in particular, tailored a perfect geometrical suit for Riemann's body of work, thereby creating what is today called the birational theory of algebraic curves.

After Cremona, considered the founder of the Italian School of algebraic geometry, came Eugenio Bertini (1846-1933), Giuseppe Veronese (1854-1917), Corrado Segre (1863-1924), and later Guido Castelnuovo (1865-1952), Federigo Enriques (1871-1946) and Francesco Severi. The latter trio's biggest contribution is the monumental classification of algebraic surfaces. Together with Gino Fano (1871-1952), moreover, they sowed the sees that would open the way to the study of higher-dimensional varieties.

In France one name stands out: that of George Halphen (1844-1889), a brilliant, multitalented mathematician who studied enumerative geometry, algebraic curves and singularities in depth, and whose work unquestionably influenced Poincaré.

\section{Poincaré's commitment to algebraic geometry}

Poincaré began as an analyst: his 1879 Thése inaugurale [16], written under the direction of Pierre Ossian Bonnet (1819-1892), was devoted to differential equations over the complex numbers and the singularities of their integrals. At the same time the influence of Charles Hermite (1822-1901) and Camille Jordan (1838-1922) prompted him to tackle the arithmetic problems at the heart of some of his early papers on quadratic and cubic forms [17-19]. Of the three fields of study mentioned in the previous section-analysis, arithmetic and geometry-Poincaré was naturally closer to the first two. Nevertheless, he made groundbreaking discoveries in geometry, as well.

I will try to present some reasons that might explain why Poincaré addressed open problems in each area.

\subsection{Theta functions}

The first problem was all the rage during the time we are considering. Riemann's key results on Abelian integrals 
showed how central these issues were for the notion of Abelian variety and its Abelian functions, of which I will now briefly review the basics.

Abelian functions are meromorphic maps on $\mathbb{C}^{g}$ admitting a lattice of periods $\Lambda=\mathbb{Z}^{2 g}$ of rank $2 g$, spanned by $2 g$ vectors in $\mathbb{C}^{g}$ that are linearly independent over $\mathbb{R}$. The building bricks to construct Abelian functions are theta functions, i.e., holomorphic maps that are quasi-periodic with respect to the lattice. Abelian functions arise as quotients of theta functions. The complex $g$-dimensional variety $A=\mathbb{C}^{g} / \Lambda$ is a group as well, whose structure is compatible with that of the variety. It is called a complex torus because, being homeomorphic to $\mathbb{R}^{2 g} / \mathbb{Z}^{2 g}$, topologically it is a torus of dimension $2 g$, that is, the product of $2 g$ circles. Whereas a complex torus of dimension one is necessarily algebraic, this is not the case as soon as $g \geq 2$, in general. Yet complex tori admitting suffciently many theta functions are algebraic, and are called Abelian varieties.

The Jacobian $J(C)$ of a smooth complex algebraic curve $C$ in projective space is a distinguished Abelian variety equipped with a theta function whose zero locus $\Theta$, called theta divisor, is a hypersurface in $J(C)$. The remarkable features of the theta divisor have to do with the geometry of $C$. For example, one basic property of $\Theta$ is that $g$ sufficiently general translates of $\Theta$ meet at $g$ ! distinct points of $A$ : this fact is nowadays formulated by saying that $\Theta$ determines a principal polarisation. The Jacobian variety's dimension equals the genus $g$ of $C$, meaning that the curve $C$, seen as a topological space, is an orientable surface and as such is homeomorphic to a surface with $g$ handles. As soon as $g \geq 4$ not every principally polarised Abelian variety is the Jacobian of a curve. Studying the Jacobian of a given curve makes it possible to understand the behaviour of the Abelian integrals related to the curve.

This theory in itself was compelling enough to entice mathematicians, especially geometers. But it is not the whole story. In the 1880 s it was gradually discovered that theta functions play a part in solving important problems in mathematical physics. One exemplary result, proved by Sonia Kovalevskaya, is that the rotation of a rigid body about a fixed point can be described using the theta functions of the Jacobian of a genus-two curve. The discovery was paramount, in that it produced another instance of a completely integrable movement of a rigid body, beside those known from work of Euler and Lagrange. Kovalevskaya was for this reason awarded the "Bordin" Grand Prix of the Académie de Paris, one of the highest recognitions a mathematician could be granted. The investigation on theta functions consequently received a big boost. Kovalevskaya herself had been looking at interesting cases producing so-called reducible theta functions, ones that can be written in terms of elliptic functions (theta functions in one variable).
That was the rich soil in which Poincaré's interest for Abelian variety thrived, and we shall see below the remarkable findings he contributed to the topic.

\subsection{Quadratic forms}

I have already mentioned that Poincaré's interest in arithmetic originated in Hermite's teachings and Jordan's work. In particular, their extension to quadratic forms in several variables with integer coefficients of Gauss's arithmetic theory (valid for the two- and three-variable case), and the corresponding theorem on the finite number of equivalence classes of unimodular transformations of variables, played a decisive role in this respect.

\subsection{Birational geometry}

The geometric approach underwent a huge expansion in the years 1860-1870. The notion of birational transformation, albeit partly contained already in Riemann, had been formalised and studied by Cremona, and became the backbone of the development of algebraic geometry. Around it grew the entire output of the mathematicians mentioned before, including Clebsch, Brill and Noether.

A further step towards the geometrisation of Riemann's ideas on algebraic curves was taken by the Italian School, notably Eugenio Bertini and Corrado Segre, concurrently with the beginning of Poincaré's scientific enterprise. The main problem at stake was passing from dimension one, i.e., curves, to higher dimensions, especially surfaces. Riemann and Clebsch mentioned the issue in passing, and the deep, but preliminary results of Noether and Pasquale del Pezzo (1859-1936) looked merely like the tip of an iceberg in an ocean almost no one had sailed across. In these same years Picard started his colossal venture, which was the first attempt to extend Riemann's theory to higherdimensional varieties, first of all surfaces. Only with the progress made in the twentieth century would this goal be achieved. Picard's idea was to make sense of integrals of multivariable algebraic differential forms and determine their periods, in the same spirit of what Abel, Jacobi and Riemann had done in one variable. This is probably one reason, if not the main driving force, that prompted Poincaré to address the issue, and develop one of his greatest gifts to us: Analysis situs, thas is, algebraic topology.

At the same time, the 1890s, Castelnuovo and Enriques were introducing a set of birational invariants aimed at classifying algebraic surfaces, which they almost perfected. The problems still to be solved, of which more later, all seemed to be essentially analytical/topological. For this reason they appealed tremendously to Poincaré, and not surprisingly his theories befitted them. 


\section{Poincaré's main contributions to algebraic geometry}

I will try to recap Poincaré's finest achievements in the various topics discussed above, and then outline a few subsequent developments inspired by his work.

\subsection{Abelian varieties and Jacobians}

The results on this subject are disseminated in a long series of papers published from 1881. It would be unreasonable to examine them here in depth, and pointless to cite them all. A substantial number of significant results is summarised in [28], a paper written upon the request of Mittag-Leffler and printed in the journal Acta Mathematica. Technical content aside, the article has an expository part that swiftly describes how the relevant theory unfolds, and also contains a useful synopsis of the most important theorems known at the time. Poincaré, apart from adding his fundamental results, contributed to render the theory coherent.

He starts out by recalling that Abelian varieties are algebraic, and that every Abelian function is the quotient of theta functions, a fact known to Riemann and Weierstrass but satisfactorily proven by Picard, Paul Appell (1855-1930) and Poincaré only later.

He then goes on to explain one of his most prominent results, known to us as the complete reducibility theorem (see also [22]). The theorem affirms that given an Abelian subvariety $A^{\prime}$ of the Abelian variety $A$, there exist an Abelian subvariety $A^{\prime \prime}$ of $A$ and an isogeny $A^{\prime} \times A^{\prime \prime} \longrightarrow A$ (an isogeny is a finite holomorphic covering map and a group homomorphism). Abelian varieties $A$ lacking trivial Abelian subvarieties ( $A$ and $\{0\}$ ) should be viewed as the simple objects in their category. It would be nice if every non-simple object were the product of simple ones: in the category of Abelian groups, for instance, the simple objects are $\mathbb{Z}$ and $\mathbb{Z}_{p}=\mathbb{Z} /(p)$ with $p$ prime. Alas, this is not the case for Abelian varieties. The complete reducibility theorem, though, guarantees that it almost works that way, meaning up to isogeny. Poincaré demonstrated this fact chiefly by arithmetic means, which is precisely what the quote from [20, p. 143] refers to. This point of view proved to be extremely fertile, and can be considered the birth of the arithmetic treatment of Abelian varieties. This theory was further developed by Gaetano Scorza (1876-1939) first, then Carlo Rosati (1876-1929), Lefschetz in the 1920s (see [1]), and is still much considered in the present (Mumford's text [12] is a 'classic' on the subject).

The complete reducibility theorem is not just a formal statement about an abstract category. Indeed, it solves the problem of finding reducible theta functions, those that can be written in terms of other theta functions with fewer variables. Particularly relevant is the reduction to the elliptic case, in which a theta function can be expressed via theta functions in one variable. This situation had been worked out by Weierstrass [who did not publish his findings but wrote them in letters to his students Kovalevskaya and Leo Königsberger (1837-1921)], and by Kovalevskaya (in three dimensions).

Poincaré then moved to consider the zeroes of theta functions. In [28] he does not give many details of the mighty result to come. This, carefully explained in [26], represents a sweeping and considerable generalisation of a crucial result of Riemann. Jacobi had proved that a curve $C$ of genus $g$ can be immersed in its Jacobian using a holomorphic map $j: C \longrightarrow J(C)$, now called the AbelJacobi map. The latter is defined up to translations by points of $J(C)$ via the integrals of differential forms that are holomorphic everywhere along $C$. Riemann had shown that the intersection between $\Theta$ and $j(C)$ consists of $g$ points, unless $\Theta$ contains $j(C)$. The Abel-Jacobi map, moreover, can be extended as follows. Denoting by $C_{d}$ the $d$-fold symmetric product of $C$ with itself, i.e., the set of unordered $d$-tuples $\left[p_{1}, \ldots, p_{d}\right]$ of points of $C$ (a $d$-dimensional variety), we obtain an Abel-Jacobi map $j_{d}:\left[p_{1}, \ldots, p_{d}\right] \in C_{d}-$ $\rightarrow j\left(p_{1}\right)+\ldots+j\left(p_{d}\right) \in J(C)$. Now set $W_{d}=j_{d}\left(C_{d}\right)$, so $W_{1}$, say, is the image of $C$ under the initial Abel-Jacobi map. Jacobi's inversion theorem, a core result in the theory of Abelian integrals, says that $W_{g}=J(C)$ and $\operatorname{dim} W_{d}=d$, for each $d<g$. Riemann had proved that $W_{g-1}$ coincides with $\Theta$ up to a translation. Poincaré demonstrated that for $d<g$ the intersection of $W_{d}$ with $d$ general translates of $\Theta$ consists of $g ! /(g-d)$ ! points. This amounts to identifying the homology class of $W_{d}$ as being $w_{d}=\theta^{g-d} /(g-d)$ !, if $\theta$ denotes the homology class of $\Theta$. As Griffiths stressed, it would not even have been possible to state such a result had Poincaré not defined the very notion of homology. To quote Griffiths once more:

As was characteristic of much of his work, several of these papers involved interaction between various branches of mathematics [11, p. 149].

I cannot conclude these remarks without mentioning a rather suggestive approach to the Schottky problem [28] and some generalisations of Abel's famous Theorem (see [22]). The Schottky problem seeks to characterise Jacobians among principally polarised Abelian variety of dimension $g>3$, and is named after Weierstrass's student Friedrich Hermann Schottky (1851-1935), who in 1888 found a partial solution when $g=4$. Poincaré pursued a clever idea of Sophus Lie (1842-1899) based on Jacobi's Inversion, implying that the divisor $\Theta$ of a Jacobian is a translate variety. In other words, it is represented parametrically by the sum of $g-1$ functions in one variable. Poincaré wrote down the local conditions for the deformations of $\Theta$ for certain Jacobians (better: certain limits of Jacobians) to be translate varieties, thus finding local 
equations for the Jacobian locus. This line of reasoning was revived eighty years later by David Mumford: his acclaimed lectures [13] influenced many in the 1980s.

Griffiths underlines [11, p. 148] that Poincaré's results (I would add Lie's, as well) on translate varieties were essential for the twentieth-century development of the theory of webs. Wilhelm Blaschke (1885-1962), ShiingShen Chern (1911-2004) and Griffiths are the biggest devotees of this still very active field of differential and algebraic geometry (see [3] and [4, §8] for Chern's firsthand testimony on its impact). Let me conclude by recalling that Poincarl'e's generalisations of Abel's theorem were a source of inspiration to Chern and Griffiths (the beautiful article [10] contains a very informative introduction about the history, with extensive references).

\subsection{Differential forms and their periods}

I have already discussed Picard's work on the integrals of algebraic differential forms in several variables. His results, proved during the nineteenth century's final two decades, were published in [15]. Poincarl'e took part in this project and contributed to it crucially. He was convinced that in order to study varieties of arbitrary dimension it is essential to understand differential forms of any given order $p$, their periods, i.e., integrals along $p$-cycles, and the relationships between them. Griffiths notes that Poincaré's achievements in algebraic topology turned out to be pivotal in bringing those ideas to life:

...we also note that Picard's great work [15] on algebraic functions of two variables would not have been possible had not Poincaré concurrently developed the necessary topological concepts [11, p. 149].

Conversely, for Poincaré algebraic varieties represented the ultimate challenge, and opened up an endless supply of examples to test his topological theories (the papers [21, 29] investigate special types of surfaces and discuss their topology).

The short note of 1886 [23] contains the so-called Poincaré Lemma, together with pointers to several applications. The result prescribes the well-known local characterisation of exact differentials $\omega$, i.e., $p$-forms that can be written as differentials of ( $p-1)$-forms: it is necessary, and sufficient, that $\omega$ be a closed form. This is a generalisation of the case $p=1$, then known, according to which for any 1-form $\omega=\sum_{1}^{n} f_{i} d x_{i}$ there exists a function $f$ such that $\omega=d f\left(f_{i}=\partial f / \partial x_{i}\right.$, for $\left.1 \leq i \leq n\right)$ if and only if $\partial f_{i} / \partial x_{j}=\partial f_{j} / \partial x_{i}$ for $1 \leq i<j \leq n$ (a necessary condition for swapping derivatives).

The most important theory is to be found in [24]. The paper defines what we now call Poincaré residue, a core notion extending the equally fundamental concept of residue of a meromorphic map at a pole. Associated to any meromorphic $n$-form $\omega$, defined on a variety $V$ with simple poles lying on a smooth $(n-1)$-subvariety $\$ \mathrm{P}, \$$ is a holomorphic $(n-1)$-form $\operatorname{Res}(\omega)$ such that $\int_{\Gamma} \omega=\int_{\gamma} \operatorname{Res}(\omega)$, where $\gamma$ is the boundary of $\Gamma$. The notion, of which Poincaré gives many applications in the same paper, plays a key role in algebraic geometry, for it may be taken as the starting point in the study of the interactions between topological and algebraic/analytical properties of varieties.

\subsection{The fundamental theorem in the theory of irregular surfaces}

The most extraordinary of Poincaré's contributions to the theory of algebraic surfaces is the so-called fundamental theorem of irregular surfaces. This elevates Poincare to the level of Castelnuovo, Enriques and Severi, among the creators of the theory.

The history of this theorem is well known, so I will just recall its salient points, and point the reader to [1] and $[2, \S 2.1]$ for further information. The problem is to extend to surfaces the notion of genus of a curve. If $C$ is a smooth curve, beside the aforementioned topological meaning of genus (the number of handles of $C$ seen as compact orientable surface, or half the first Betti number of $C$, that is, half the rank of the first homology group of $C)$, there are two more, equally important, descriptions. The first, uncovered by Riemann, is that the genus equals the maximum number of linearly independent holomorphic 1-forms on $C$. The second, due to Clebsch, is a stepping stone in the algebro-geometrical understanding of Riemann's theory as developed by Brill, Noether and the Italian scholars. If we realise $C$ as a curve in the complex projective plane $\mathbb{P}^{2}$, it will typically have singularities, the simplest of which can be nodes, i.e., crossings of two smooth branches with different tangent lines. It can be proved that every curve can be realised as a plane curve of given degree with a certain number of distinct nodes.

Allow me a little detour. Kronecker had a sense of the previous fact already in 1859 , when he mentioned it in a letter to Klein [9, vol. 2, p. 540]. The result was part of the conventional wisdom, but as pointed out in $[9$, vol. 2, p. 550] "it is fully proved in a note by Poincaré" (cf. [25]). Here we see how Enriques, a forebear of Italian algebraic geometry, has to admit that the first rigorous proof of this key property goes back to Poincaré, who-another noteworthy fact- did not disdain matters that he knew would not bring him any glory. While it would be interesting to discuss the contents of this short note more in detail, this is not the right place for a technical debate. 
Now, back to our point, if $C$ is a plane curve of degree $d$ with $\delta$ nodes, its genus $g$ obeys Clebsch's Formula $g=$ $\left(\begin{array}{c}d-1 \\ 2\end{array}\right)-\delta$. This relation has a concrete meaning. If $f(x, y)=0$ is the equation describing $C$, the meromorphic 1 -form $\omega=\frac{g(x, y)}{\partial f}(x, y) d x$, with $g(x, y)$ a polynomial ( $\omega$, a posteriori, is nothing else than Poincaré's residue for the 2-form $\frac{g(x, y)}{f(x, y)} d x \wedge d y$ ), is holomorphic on $C$ if and only if $g(x, y)=0$ is a curve of degree $d^{\prime} \leq d-3$ that is adjoint to $C$, i.e., a curve passing through all $\delta$ nodes. Furthermore, every holomorphic 1 -form on $C$ arises in this way. Since the genus $g$ is the largest number of linearly independent holomorphic 1-forms on $C$, it also turns out to be the maximum number of linearly independent adjoint curves to $C$ of degree $d-3$.

In order to define the genus of a surface $S$, then, one can adapt these various descriptions of the unique notion of genus of a curve. However, this will lead to different genera for $S$. First of all one can consider the maximum number of linearly independent holomorphic 2-forms on $S$. This gives the so-called geometric genus of $S$, denoted by $p_{g}$ and introduced by Clebsch in the 1870s. If we think of the surface in $\mathbb{P}^{3}$ of degree $d$ with simplest-possible singularities (a curve $\Gamma$ of double points), $p_{g}$ is indeed the maximum number of adjoint surfaces (through $\Gamma$ ) of degree $d-4$, as in the case of curves. More or less around the same time Arthur Cayley (1821-1895), Noether and Hieronymous Georg Zeuthen (1839-1920) devised another invariant $p_{a}$, the arithmetic genus of $S$. This is the virtual maximum number (in a sense I will not explain here, see $[2, \S 2.1]$ ) of linearly independent adjoint surfaces of degree $d$-4. We always have $p_{g} \geq p_{a}$, and there are situations in which the inequality is strict. The invariant $q=p_{g}-p_{a}$ was named irregularity. We shall indicate it by $q_{a}$ and call it arithmetic irregularity. Next, one may consider the maximum number of linearly independent holomorphic 1-forms, giving the analytic irregularity $q_{\text {an }}$ defined by Picard. One can then take half the rank of the first homology group of $C$, the topological irregularity $q_{t}$ of Picard and Poincaré. There is, eventually, one further and very subtle invariant having an exquisite algebro-geometrical flavour, namely the maximal dimension of a family of curves on $S$ that are not linearly equivalent (two curves on $S$ are linearly equivalent if, possibly adding to both a third curve, one is the curve of zeroes, the other the curve of poles of a single rational map on $S$ ). This last invariant is denoted $q_{g}$ and we call it the geometric irregularity of $S$. At the turn of the nineteenth century one crucial question within the classification of surfaces was to determine the relationships among these quantities.

The period 1890-1910 was fundamental for sorting out this issue. Here is a short summary of the main steps in this direction (more information can be found in [33, ch. VII and the Appendix by Mumford]):
(i) (Humbert 1893)
If $q_{g}>0$, then $q_{\text {an }}>0$.
(ii) (Enriques 1899)
$q_{g}>0$ implies $q_{\mathrm{a}}>0$.
(iii) (Enriques 1901)
$q_{t}>0$ forces $q_{\mathrm{g}}>0$.
(iv) (Severi 1905)
$q_{\mathrm{an}} \leq q_{\mathrm{a}}$.
(v) (Enriques 1904)
$q_{a}=q_{g}$.

The 1904 paper by Enriques [7] contained a mistake, discovered by Severi only in 1921. This was the beginning of a long and unpleasant dispute between the two. Since much has been written about this, I will not add more [8, ch. IX, §6], [6]. In 1905 Castelnuovo and Severi independently proved that $q_{a}=q_{g}=q_{\text {an }}=q_{t}$ relying on Enriques's result. This is known as the fundamental theorem of irregular surfaces.

Poincaré, who had an evident interest in the matter because of the theorem's topological consequences, wrote a correct proof for this fact in [30]. The analytic/topological argument was truly innovative, it was entirely independent of Enriques's geometrical approach, and it exposed the concealed nature of some essential aspects. It is brilliantly explained in Griffiths's article [11], and due to its technicality we shall not discuss it further. It will suffice to mention that in [30] Poincaré introduced several valuable notions, for instance normal functions: in a nutshell, a normal function accounts for the variation of an Abelian variety within a family (in his case, a Jacobian varying in the family of plane sections of a surface). Poincaré succeeded in determining the geometric structure for the case in question, and infer from this the fundamental theorem of irregular surfaces.

Despite Enriques's efforts to amend his error (see [8] and Mumford's recent article [14], advocating for a retrieval of Enriques's intuitions), Poincaré's was the only available proof of the theorem. About 60 years had to go by before modern sophisticated techniques (sheaves, cohomology, Serre's duality, plus the De Rham, Dolbeault and Hodge theorems) made it possible to simplify his techniques. On the other hand normal functions - though in a more general form than the original-were key to attaining those techniques, and this is true even at present. The so-called Lefschetz $(1,1)$ theorem, and more generally Hodge theory [named after William Vallance Douglas Hodge (1903-1975)] are exemplary in this sense.

\subsection{Arithmetic geometry}

As I indicated previously, Poincaré started quite early to look at Diophantine problems and arithmetic geometry. His first short papers $[17,18]$ on quadratic forms, for instance, date to 1879 . I would like spend a few words on what is 
commonly accepted as his foremost contribution to arithmetic geometry, i.e., the terrific memoir [27] (by the way his final publication on the topic). The problem examined is the following: given a plane curve of equation $f(x, y)=0$, with $f$ a polynomial with coefficients in $\mathbb{Q}$, determine its rational points, those whose coordinates are all rational numbers. Poincaré set off from the birational algebraic geometry of Cremona and Noether, and noted that the question was invariant under birational transformations of the plane defined over $\mathbb{Q}$, i.e., for generically invertible transformations of the type $(x, y) \longrightarrow(\phi(x, y), \psi(x, y))$, where $\phi, \psi$ are rational functions of $(x, y)$ over $\mathbb{Q}$. As the genus of a curve is invariant under such maps, Poincaré was led to consider how the genus of $f(x, y)=0$ affected the solution of the problem. After examining rational curves (genus 0), he concentrated on the first interestingand hard-case, that of elliptic curves (genus 1). For this Poincare used the fact that an elliptic curve $C$, seen over the complex field, is isomorphic to its Jacobian under the Abel-Jacobi map. Thus the set of its rational points corresponds to a subgroup of the Jacobian whose rank, called by Poincare the rank of the curve, is an invariant. Poincaré developed a daring method that employed elliptic functions to compute the rank, which he implicitly assumed to be finite. The paper ends with a few questions on the highergenus case.

That a rational elliptic curve has finite rank was proved by Louis Joel Mordell (1888-1972) in 1922, relying on ideas of Poincaré. Weil extended that result in his 1928 $\mathrm{Ph} . \mathrm{D}$. thesis, while introducing totally novel concepts, and proved that for curves of genus larger than one, as well, the rank of the rational points of the Jacobian is finite. It is worth remarking that in his autobiography [32] Weil attributes to Poincare the problem he solved in his dissertation. Today this is known as the Mordell-Weil theorem, and lies at the heart of many subsequent developments. Tellingly, one such development is Gerd Faltings's solution to the Mordell conjecture, which states that a curve of genus $g>1$ over a number field $\mathbb{K}$ has at most finitely many rational points over $\mathbb{K}$. On this account Faltings received the Fields Medal in 1986. On the other hand many a problem tackled by Poincaré in [27] remain unsolved. The main difficulty is probably that of finding an efficient computational method to compute the rank of an elliptic curve.

\section{Conclusions}

Poincaré's vision of mathematics, and more broadly of science, culture, and life, was too far-reaching for us to classify his work and confine it in any feasible way. It is therefore a difficult task to specify what might be his legacy in algebraic geometry only, without being forced to trespass into complex analysis, topology or number theory.

The 100 years that have passed since his premature death have more than confirmed but one thing: that a good deal of the mathematics proliferated since Poincaréalgebraic geometry especially — can be traced back to ideas he introduced, more or less explicitly. Here I have attempted to sum up, obviously in a non-exhaustive, but partial and incomplete manner, his principal contributions to algebraic geometry, thereby following the many trails that he left us, and that have been pivotal for the ensuing developments.

Translated from the Italian by Simon G. Chiossi

\section{Appendix: A friend of the Circolo}

Poincaré held Italian mathematics in high esteem, particularly the experiment carried out by the founder of the Circolo matematico di Palermo, Giovan Battista Guccia. In an report that he wrote about the 1908 International Congress of Mathematicians in Rome, published in the Paris newspaper, Le Temps, he mentioned him specifically: "Guccia, who did fine work in geometry and founded in Palermo an international mathematical society and one of the mathematical journals most widely read in the whole world." In addition to the scientific solidarity and the complicity of Poincare in helping the Circolo achieve a dimension that was genuinely international that linked the two mathematicians, there was also a cordial friendship. One sign of this was the trip that the French mathematician made to Palermo in the company of his cousin Raymond Poincaré, the future president of the French Republic. It was on this occasion that Henri gave the lecture entitled 'Quelques remarques sur les groupes continus', which constituted an important step forward in the development of the theory of Lie groups.

Poincaré became a member of the Palermo Circolo in 1890, but his collaboration with the Circolo's journal, the Rendiconti, had begun two years earlier, with the publication of the paper 'Sur une propriété des fonctions analytiques.' Later the Rendiconti published articles that were fundamental for the evolution of mathematical physics and algebraic topology, such as 'Sur les équations de la Physique Mathématique' (1894) and 'Complément à l'analysis situs' (1899). Together with the eleven works that appeared after the beginning of the new century, the Rendiconti became the journal in which Poincare published the greatest number of articles. Of these, the most famous is perhaps 'Sur la dynamiques de l'électron' (1906), which represents Poincaré's most important contribution to the theory of relativity. In 1908 the Circolo was the first to print the 'Avenir des mathématiques', the text of the 
lecture prepared by Poincaré for the Rome Congress. Poincaré's special tie to the Circolo, once again demonstrated by the publication in June 1912 (3 months before his death) of the article 'Sur un théorème de géométrie' regarding the three-body problem and considered the French mathematician's testament. The letter to Guccia that accompanied that article (quoted in the book by Aldo Brigaglia and Guido Masotto, Il Circolo Matematico di Palermo, Dedalo, Bari, 1982) is a further testimonial to their friendship: ('My dear friend ... what embarrasses me is that I am obliged to put many figures, precisely because I have not been able to arrive to a general rule. ... Tell me, I beg you, what you think and what you advise me to do.'

\section{References}

1. Brigaglia, A., Ciliberto, C.: La geometria algebrica italiana tra le due guerre mondiali. In: Di Sieno, S., Guerraggio, A., Nastasi, P. (eds.) La matematica italiana dopo l'unitàà, Gli anni tra le due guerre mondiali, pp. 185-320. Marcos y Marcos, Italy (1998)

2. Brigaglia, A., Ciliberto, C., Pedrini, C.: The Italian School of Algebraic Geometry and Abel's Legacy. In: Laudal, O.A., Piene, R., (eds.) Legacy of N. H. Abel, The bicentennial, Oslo, 2002. pp. 295-348. Springer, Berlin (2004)

3. Chern, S.S.: A mathematician and his mathematical work: selected papers of S.S. Chern, edited by S.Y. Cheng, P. Li, G. Tian. World Scientific, Singapore (1996)

4. Chern, S.S.: A Summary of My Scientific Life and Work, in [3], pp. 61-71 (1978)

5. Ciliberto, C.: L'impronta di Eulero sulla teoria delle funzioni ellittiche. Lettera Matematica Pristem 66(67), 53-60 (2008)

6. Ciliberto, C.: Enriques e Severi: collaborazioni e contrasti, Pubblicazioni del centro Studi Enriques, 4. In: Pompeo Faracovi, O., (ed.) Atti Convegno "Enriques e Severi, matematici a confronto nella cultura del novecento", Livorno, 24-25 ottobre 2002, pp. 29-49

7. Enriques, F.: Sulla proprietàà caratteristica delle superficie algebriche irregolari. Rend. Accad. Sci. Bologna 11, 5-13 (1905)

8. Enriques, F.: Le superficie algebriche. Zanichelli, Bologna (1949)

9. Enriques, F., Chisini O.: Lezioni sulla teoria geometrica delle equazioni e delle funzioni algebriche, vol. 4. Zanichelli, Bologna (1915-1934)

10. Griffiths, $\mathrm{Ph} .:$ Variations on a theorem of Abel. Inventiones math, 35, 321-390 (1976)

11. Griffiths, Ph.: Poincaré and algebraic geometry. Bull. Amer. Math. Soc. 6(2), 147-159 (1982)

12. Mumford, D.: Abelian Varieties. Oxford University Press, Oxford (1970)

13. Mumford, D.: Tata Lectures on Theta: Jacobian theta functions and differential equations. Springer, Berlin (1984)

14. Mumford, D.: Intuition and Rigor and Enrique's Quest: a modern analysis. Notices Am. Math. Soc. 58(2), 250-260 (2011)

15. Picard, E., Simart, G.: Théorie des fonctions algébriques de deux variables independentes. Gauthier-Villars, Paris (1897-1906)

16. Poincaré, H.: Sur les propriétés des fonctions définies par des équations aux différences partielles. Gauthier-Villars, Paris (1879)
17. Poincaré, H.: Sur les formes quadratiques. Comptes rendus hebdomadaires de l'Académie des sciences 89, 87-89 (1879)

18. Poincaré, H.: Sur quelques propriétés des formes quadratiques. Comptes rendus hebdomadaires de l'Académie des sciences 89, 344-346 (1879)

19. Poincaré, H.: Sur les formes cubiques ternaires. Comptes rendus hebdomadaires de l'Académie des sciences 90, 1336-1339 (1880)

20. Poincaré, H.: Sur la réduction des intégrales abéliennes. Bulletin de la SMF 12, 124-143 (1884)

21. Poincaré, H.: Sur les intégrales de différentielles totales. Comptes rendus hebdomadaires de l'Académie des sciences 99, 1145-1147 (1884)

22. Poincaré, H (1886) Sur les fonctions abéliennes. Am. J. Math. 8, 289-342

23. Poincaré, H.: Sur les résidus des intégrales doubles. Comptes rendus hebdomadaires de l'Académie des sciences 102, 202-204 (1886)

24. Poincaré, H.: Sur les résidus des intégrales doubles. Acta Mathematica 9, 321-380 (1887)

25. Poincaré, H.: Sur les transformations birationnelles des courbes algébriques. Comptes rendus hebdomadaires de l'Académie des sciences 116, 18-23 (1893)

26. Poincaré, H.: Remarques diverses sur les fonctions abéliennes, 5th series. J. de Math. 1, 219-314 (1895)

27. Poincaré, H.: Sur les propriétés arithmétiques des courbes algébriques. Journal de Mathématiques pures et appliquées 7, 161-233 (1901)

28. Poincaré, H.: Sur les fonctions abéliennes. Acta Mathematica 26 , 43-98 (1902)

29. Poincaré, H.: Sur certaines surfaces algébriques; troisième complément àà l' 'Analysis sitÛs. Bulletin de la Société mathématique de France 30, 49-70 (1902)

30. Poincaré, H.: Sur les courbes tracèes sur les surfaces algèbriques. Ann. Ècole. Norm. Sup. 27, 3 (1910)

31. Poincaré, H.: Analyse de travaux scientifiques de Henri Poincaré fait par lui-même. Acta Mathematica 38, 1-135 (1921)

32. Weil, A.: The Apprenticeship of a Mathematician. Birkhäuser, Basel (1992)

33. Zariski, O.: Algebraic Surfaces, second supplemented edition. Ergebnisse der Math., vol. 61. Springer, Berlin (1971)

\section{Author Biography}

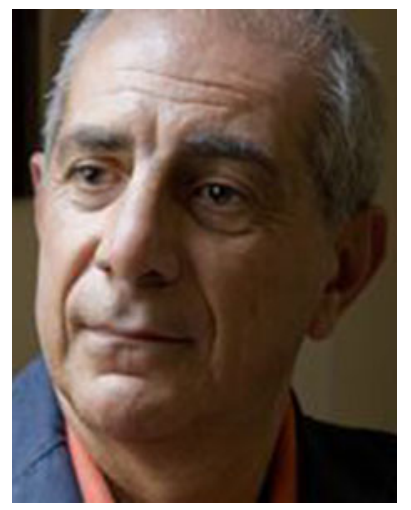

Ciro Ciliberto Teaches Geometry at the University of Rome Tor Vergata. His main scientific interests are in Algebraic Geometry. 